RESEÑAS BIBLIOGRÁFICAS 



\title{
"FALSAS PROMESAS. SISTEMA DE PREVISIÓN SOCIAL Y RÉGIMEN DE ACUMULACIÓN"
}

\author{
de Rubén Lo Vuolo y \\ Laura Goldberg (2006) \\ Miño y Dávila, Buenos Aires.
}

por Oscar Núñez

El libro Falsas Promesas. Sistema de previsión social y régimen de acumulación, de los Licenciados Rubén Lo Vuolo y Laura Goldberg, cuya publicación es de unos meses anterior a la sanción de la última reforma de principios de 2007, intenta fundamentar los motivos por los cuales no han funcionado las recetas aconsejadas desde hace algunas décadas, por organismos internacionales e incorporados a las diferentes propuestas de reformas que, en definitiva, sivieron de base para las modificaciones introducidas en la Seguridad Social en 1994.

También pone de relieve el esfuerzo que, actualmente, dichos organismos hacen en justificar su accionar fortificando solapadamente un modelo, a través de una nueva reforma. En el caso específico de las reformas de los sistemas de previsión social, los autores, recorren el problema observándolos desde distintas perspectivas. En el orden económico, los cambios en el patrón dis- tributivo, y los cambios en relación con los procesos de reproducción del capital. En el orden político, los impactos en la definición de la ciudadanía.

Comienzan con un análisis de los inicios caóticos de las instituciones de la seguridad social para llegar a nuestros días, desde su construcción, en base a esquemas diversos y superpuestos, pasando por el "primer régimen de previsión social" en la segunda mitad de la década del 40 , en el cual la distribución de los derechos se basaba en el estatus y guardaba una lógica particularista, llegando a la reforma del 1968, la cual produjo una estandarización de los criterios de elegibilidad, de los recursos contributivos y del modo de cálculo de los beneficios.

Destacan que, en dicha reforma, se revirtió la distribución de accesos anterior basada en el estatus y se redefinió la forma del patrón de distribución de derechos hacia uno basado en la contribución, estrechamente ligado a los 
ingresos de los trabajadores. Este esquema permaneció hasta la reforma del 1994.

Respecto del funcionamiento del sistema, considera que se originaron ciertas brechas:

- La primera tiene que ver con la cobertura real de la población activa. Si bien en la cobertura legal era universal, en la práctica, no.

- Las condiciones de autofinanciamiento, con la estandarización y homogeneización de las normas, no se lograron ya que se fue incorporando paulatinamente y en forma creciente, el financiamiento por rentas generales.

- Respecto a La movilidad de los haberes, la legislación ordenaba preservar su valor real en función de los salarios. En la práctica no se pudo lograr.

Con estas brechas, en un contexto de crisis como la Hiperinflación de 1989-90, termina por deslegitimar al Sistema Nacional de Previsión Social (SNPS).

Los autores analizan los motivos que llevaron a provocar la reforma, inspirada en los modelos promocionados por la Nueva Ortodoxia Previsional (NOP) y los actores que intervinieron. Luego realizan un análisis de las consecuencias del nuevo sistema.

Los resultados de la reforma previsional de 1994 contrastan enormemente con las proyecciones optimistas. La importancia de las brechas alcanzadas responde a la lógica de funcionamiento de los nuevos arreglos operativos en el particular ambiente económico, político y social del país.

En un pormenorizado análisis demuestran que la reforma profundizó la crisis en aquellos aspectos que pretendía corregir:

- Se profundizó el desequilibrio financiero y las cuentas fiscales

- Se deterioró las condiciones de vida de los pasivos.

- Creció el patrón distributivo regresivo.

- Cayó la cobertura
- Se incrementó la fragmentación normativa e institucional.

- No produjo los cambios macroeconómicos en el ahorro e inversión.

- Creció el costo en los servicios.

Lo Vuolo y Goldberg reconocen que la multiplicidad y profundidad de los problemas producidos no pueden revertirse con medidas aisladas, sino que reclaman una aproximación sistémica que permita identificar tres cuestiones centrales:

- La necesidad de revertir la tendencia a profundizar el patrón regresivo del sistema.

- El cambio de su relación con el proceso de acumulación de capital.

- La sustitución de un sistema que construye ciudadanía patrimonial por otro que se asiente sobre una ciudadanía de acceso a derechos más igualitarios.

El Banco Mundial (BM) considerado el principal responsable de la reforma, ha planteado la necesidad de una "revisión" del modelo de la NOP. Argumentan que los problemas que presentan los nuevos sistemas de previsión social se deben a que los esfuerzos de reforma se concentraron en mejorar la "salud fiscal" de los sistemas y en la regulación gubernamental de los mismos. Sin embargo no se preocuparon por evaluar si las reformas impactarían beneficiosamente a los participantes del sistema.

Con certeza afirman los autores que entre el nuevo modelo planteado por BM, incluye un esquema de gerenciamiento del riesgo social basado en un esquema de distribución de riesgo en vez de distribución de ingresos y plantea un sistema de protección social como etapas evolutivas. La visión integra cuatro elementos:

- Desaconseja "saltar" desde sistemas de reparto a sistemas de capitalización, sino que 
puede pensarse en una transición a través de cuentas nocionales;

- Admite como no recomendable que los sistemas de capitalización sean permanentemente obligatorios;

- Esta variante sobre el pilar de capitalización se complementa con nuevas recomendaciones respecto a atender la pobreza en la edad pasiva;

- Manifiestan la conveniencia de subsidiar la incorporación obligatoria a los seguros colectivos.

Ahora, el BM se decide por un beneficio universal y uniforme para atender a la pobreza de los mayores, no obstante de las innumerables críticas que había realizado en 1994 a este criterio. Sin embargo, si bien aparenta preocupación en este sentido, continúa fortaleciendo el inevitable pilar de prevención de la pobreza para que no reste fondos ni protagonismo al de capitalización financiera de aportes, único objetivo real del B.M.

Estamos de acuerdo en que la prioridad debe estar situada en el diseño de un beneficio básico universal que prevenga la pobreza y todos los otros pilares del sistema de previsión social deberían subordinarse a este objetivo prioritario. Pero con prolijidad y prudencia los autores avanzan un poco más en sus afirmaciones. Analizan contemporáneamente, una alternativa intermedia entre las que buscan resolver el problema con cambios en los parámetros operativos de los sistemas de reparto y estrategia de reformas más o menos integrales que trasfieren parte de los aportes a cuentas individuales de capitalización: Los Sistemas de Cuentas Nocionales. Indagan su aplicación en distintos países y principalmente en Suecia y analizan la posibilidad de implementación en nuestro país. Concluye en ese sentido que en Argentina no resultaría adecuado establecer un sistema de Cuentas Nocionales (SCN) como primer pilar debido a la indeterminación del haber resultante. Los indicadores de distribución del ingreso y de incapacidad de aporte del país no permiten que el SCN garantice la cobertura universal ni el acceso a niveles mínimos de ingreso.

Los expertos abren el debate presentando lineamientos que merecen ser tomados en cuenta. Un único sistema público obligatorio compuesto por dos pilares:

- Un pilar básico que afronte un beneficio universal, uniforme e incondicional.

- Otro, contributivo obligatorio cuyos beneficios estén vinculados a los aportes y que se financien con un mecanismo de reparto, de modo que permita distribuir los riesgos individuales.

Entendemos, dicen, “...que el modo menos traumático de cambiar la situación actual es mediante el diseño de un esquema de reparto basado en cuentas nocionales que observan las actuales cuentas individuales de capitalización financiera administradas de forma privada...".

- Finalmente quedaría habilitada la opción voluntaria de contratar Seguros de Retiros privados, fuera del alcance del sistema previsional público.

Esto contribuiría a superar los límites que imponen la informalidad y el clientelismo a la construcción de un sistema de protección social con cobertura universal y capaz de crear una ciudadanía igualitaria. Reduciría asimismo, las complejidades que actualmente plantea la política de previsión social, atendiendo al interés general de la manera más directa posible.

Coincidiendo con el diagnóstico y el análisis realizado por los autores, es necesario destacar algunos aspectos que parecen cruciales a la hora de resolver problemas sociales y que en el libro, se hacen hincapié: 
1- La falta de información que disponían los actores interesados en el tema a la hora de discutir las diversas alternativas y la falta de un liderazgo capaz de coordinar las distintas propuestas presentadas. Argentina tiene una larga historia de reformas previsionales en la cual a primado el criterio de la "imposición". Evidentemente lo más complejo de cambiar son los intereses particulares de algunos grupos se disfrazan de medidas justas y técnicamente eficientes. Esos intereses sectoriales se deben limar con mecanismos muy poco usados en la Argentina como es el "Consenso", el "Pacto", en el buen sentido de la palabra, en definitiva en un "Contrato Social", en el cual deben participar todos los actores sociales que tengan que ver, en este caso, con la Seguridad Social, con voz y voto. Esto es, realizar una verdadera "Convención Social" para la reforma de la Seguridad Social. La pregunta a responder antes de decidir el régimen que nos cubrirá y alcanzará a generaciones futuras es la siguiente: ¿Qué tipo de Seguridad Social queremos para nuestro ciudadanos: Asistencialista, Contributiva o Mixta? Grandes países, con amplia trayectoria en materia de Seguridad Social, (España, Alemania, Suecia, Italia y en nuestro continente, Brasil, Bolivia, Colombia), han invertido años en la búsqueda de un consenso social para implantar un Sistema de Seguridad Social acorde con sus necesidades. No debemos olvidar que la Seguridad Social es una herramienta fundamental para la distribución del ingreso y para delinear en el tiempo diversas políticas sociales, por lo tanto debe ser tratada con la importancia y el fundamento que se merece si se quieren "prevenir" peores crisis sociales. La Seguridad Social debe ingresar, de una vez y para siempre, en la agenda política de un proyecto a largo plazo.
2- Otro aspecto importante en el tema es considerar a la Seguridad Social como un verdadero Sistema. La diversidad y cantidad de regímenes previsionales vigentes en todo el país, los problemas habituales que atiende la Seguridad Social tales como: vejez, invalidez, salud, cobertura asistencial, considerados en forma aislada, como sistemas totalmente separados y otras cuestiones sociales no consideradas o nuevos requerimientos sociales surgidos del propio desarrollo social de la época, que no encuentran una cobertura adecuada y que asumen actualmente, en forma creciente, complejas dimensiones no abordadas por otras disciplinas. Todo esto debería ser considerado, de alguna forma, en ese gran sistema se seguridad social que se debe consensuar.

El libro permite al lector comprender que los modelos que se experimentaron en otros contextos sociales pueden ser tenidos en cuenta como marco de referencia pero no pueden ser replicarlos íntegramente. Nos marca la importancia que posé la cobertura básica universal en las prestaciones como herramienta de redistribución de los ingresos y contención social y nos brinda alternativas esperanzadoras respectos a las salidas que posee el Sistema de Seguridad Social en la Argentina para darle una sostenibilidad financiera y social en el tiempo y principalmente evitar que las políticas en materia de Seguridad Social sea una herramienta de ajuste. Aunque, muchas de estas alternativas no fueron tomadas en cuenta en la última reforma, sirven para continuar el debate para una reforma definitiva y sostenible. 